\title{
Limit of the buoyancy ratio in Boussinesq approximation for double-diffusive convection in binary mixture
}

\author{
Saber Hamimid,, ${ }^{7, a)}$ (D) Messaoud Guellal, ${ }^{1, b)}$ (D) and Madiha Bouafia ${ }^{2, c)}$ (iD \\ 'Laboratoire de Génie des Procédés Chimiques, Université Ferhat Abbas Sétif 1, Sétif 19000, Algeria \\ ${ }^{2}$ Laboratoire de Mécanique et Energétique d'Evry, Université d'Evry, 91020 Evry Cedex, France
}

\footnotetext{
a) Author to whom correspondence should be addressed: sa hamimid@yahoo.fr

b) mguellal@univ-setif.dz

c) madiha.bouafia@univ-evry.fr
}

\begin{abstract}
This paper deals with a mathematical and numerical investigation of double-diffusive natural convective heat and mass transfer in a cavity filled with Newtonian fluid with significant density and mass diffusivity changes. In such a situation, the assumption of the Boussinesq approximation is not justified, and an appropriate model based on a set of Low Mach Number equations is used. The active parts of two vertical walls of the cavity are maintained at fixed but different temperatures and concentrations, while the other two walls, as well as inactive areas of the sidewalls, are considered to be adiabatic and impermeable to mass transfer. The coupled momentum, energy, and solute transfer equations in binary mixtures of ideal gases are solved through a global iterative procedure based on the finite volume methods in the context of the low Mach number approximation. The study includes the effect of the buoyancy ratio $\mathrm{N}$ with the aim to find its application limit in the Boussinesq conditions. The results show that if we use the Boussinesq approximation to study double-diffusive convection, the value of parameter $\mathrm{N}$ must be between -6 and 27 .
\end{abstract}

\section{NOMENCLATURE}

$C$
$c_{p}$
$D$
$g$
$H$
$\mathrm{k}$
$L e=\alpha / D$
$M$
$M^{*}$
$\boldsymbol{n}$
$N$
$N u$
$p$
$\bar{p}$
$\operatorname{Pr}=\nu / \alpha$
$R a=g \beta \Delta T H^{3} /(\nu \alpha)$
$S h$

concentration, $\mathrm{kg} / \mathrm{kg}$ specific heat

binary mass diffusion coefficient, $\mathrm{m}^{2} / \mathrm{s}$ gravitational acceleration, $\mathrm{m} / \mathrm{s}^{2}$

height of the enclosure, $\mathrm{m}$

thermal conductivity, $\mathrm{W} / \mathrm{m} \mathrm{K}$

Lewis number

molecular weight, $\mathrm{kg} / \mathrm{kmol}$

molecular weight ratio, $M^{*}=M_{1} / M_{2}$

unit outward normal to surface $d S$

Buoyancy ratio

convective Nusselt number

dynamic pressure, $\mathrm{Pa}$

mean thermodynamic pressure, $\mathrm{Pa}$

Prandtl number

Rayleigh number

local Sherwood number

$\begin{array}{ll}t & \text { time, } s \\ T & \text { temperature, } \mathrm{K} \\ U, V & \text { dimensionless velocity-components } \\ \mathrm{u}, \mathrm{v} & \text { velocity-components, } m / s \\ W & \text { mass fraction } \\ X, Y & \text { dimensionless coordinates }\end{array}$

\section{GREEK SYMBOLS}

$$
\alpha=k / \rho . c_{p}
$$

$\varepsilon_{T}$

$\varepsilon_{m}$

$\nu$

$\Pi=\left(p-\bar{p}+\rho_{0} g y\right) / \rho_{0}\left(\alpha_{0} / H\right)^{2}$ thermal diffusivity thermal expansion coefficient, $\mathrm{K}^{-1}$

non-Boussinesq thermal parameter $\varepsilon_{T}=\Delta T / 2 T_{0}$ non-Boussinesq solutal parameter $\varepsilon_{m}=\frac{\Delta C_{1}\left(1-M^{*}\right)}{M^{*}+C_{10}\left(1-M^{*}\right)}$ kinematic viscosity, $\mathrm{m}^{2} / \mathrm{s}$ dimensionless dynamic pressure 


\section{SUBSCRIPTS}

$\begin{array}{ll}\text { avg } & \text { average value } \\ B S & \text { Boussinesq model } \\ c & \text { cold } \\ \text { CP } & \text { constant properties } \\ h & \text { hot } \\ \text { max } & \text { maximum value } \\ \text { mid } & \text { midplane } \\ \text { min } & \text { minimum value } \\ 0 & \text { reference state } \\ 1 & \text { for gas } \\ 2 & \text { for air } \\ * & \text { dimensionless parameters }\end{array}$

\section{INTRODUCTION}

Compressible low-Mach number flows of double diffusive convection appear frequently in nature and industrial processes such as atmospheric and oceanographic flows, combustion, vapor deposition, and natural gas storage. The compressible low-Mach number flow $(M a \ll 1)$ is defined as such when pressure variations are small, but temperature/concentration (and hence density) gradients are large.

Natural convection in enclosures filled with binary mixtures due to combined thermal and solutal body forces has been investigated extensively both experimentally and numerically and has received increasing attention. Over the past decades, a considerable body of research has thus been conducted in this field, both experimentally and numerically.

The greatest portion of the more pertinent binary gas literature has focused on analytical and numerical investigations of natural convection due to horizontal temperature and concentration gradients. $^{6-12}$

With the exception of some works using the non-Boussinesq model, ${ }^{13-17}$ it appears that the Boussinesq approximation was used in both cases of aiding and opposing buoyancy forces, which states that variations of density in the equations of motion can safely be ignored everywhere except in its association with the external force. The approximation is well justified in the case of incompressible fluids.

Ferziger and Peric ${ }^{18}$ assert that the use of the Boussinesq approximation in the Navier-Stokes equations introduces errors of the order of $1 \%$ if the temperature differences are less than $2{ }^{\circ} \mathrm{C}$ for water. These errors are led to augment for more important difference of temperature and can lead to qualitatively wrong resolutions.

The effect of buoyancy ratio on the flow structure was investigated numerically for a binary mixture gas in a rectangular enclosure by many authors. ${ }^{19-23}$

In most of the works studying double-diffusive convection, a wide range of the buoyancy ratio $N$ was considered. The question that arises is whether the buoyancy ratio $N$ has a limit when using the Boussinesq approximation. Such a ratio $N$ denotes the relative strengths of the thermal and solutal buoyancy forces, and it is based on the concentration and temperature differences between left and right vertical walls. In this study, we will try to answer this question by taking into account the variation of the thermophysical properties of the studied fluid.

When the fluids are compressible, the equations governing the system become quite complicated. In such situations, all assumptions used to justify the Boussinesq approximation fail and a different modeling approach is required, one that accounts for realistic nonlinear fluid property variations. The Low Mach Number approximation $(\mathrm{Ma} \ll 1)$ suggested by Paolucci ${ }^{24}$ was developed in our previous works for natural convection; natural convection coupled with surface radiation and combined natural convection-volumetric radiation. ${ }^{25-28}$ This approach is used in this study to solve the momentum, heat, and solute equations, which lead to take into account fluid flows under large temperature/concentration gradients and variable transport coefficients.

The purpose of the present paper is twofold: the first is to develop a mathematical model describing the double diffusive convection under non-Boussinesq conditions and the other is to investigate the limit of the buoyancy ratio of the double-diffusive convection in vertical enclosures under the Boussinesq approximation.

\section{MATHEMATICAL FORMULATION}

The aim of this section is to provide the governing equations to be solved by the low-Mach number solver for the double-diffusive convection. The low-Mach number approximation is carried out, enabling the full low-Mach number system of equations to be written.

The geometry investigated as shown in Fig. 1 is an enclosure filled with a gas mixture in which natural convection is driven by thermal and solutal gradients. The left wall is kept at high temperature $\left(T_{h}\right)$ and high concentration $\left(C_{1 h}\right)$, the right wall at low temperature $\left(T_{c}\right)$ and low concentration $\left(C_{1 c}\right)$ and the bottom and top walls are adiabatic and impermeable. No-slip conditions are applied on all boundaries. The kinematic viscosity, the thermal diffusivity, and the concentration diffusivity are determined by the properties of this binary gas mixture.

\section{A. Governing equations}

Since we are interested in the case of large thermal and solutal gradients, the low-Mach-number equations are adopted ${ }^{24,27}$ in order to describe such a flow.



FIG. 1. The flow configuration and coordinate system. 
Stemming from the compressible Navier-Stokes equations, the Low Mach Number (LMN) approximation constitutes an important numerical problem for low speed compressible flows and has the advantage that it presents the same mathematical structure as the incompressible Navier-Stokes equations.

In this approximation, and as shown in Ref. 24, the total pressure is divided into two components: a mean thermodynamic pressure $\bar{p}(t)$ which is spatially uniform and depends only on time and a dynamic pressure $p_{d y n}$. Since for Low Mach number $(\mathrm{Ma} \ll 1)$, the thermodynamic pressure is very high compared to the dynamic pressure, and this decomposition leads to elimination of the acoustic waves while large variations of density with temperature and concentration are allowed.

The conservation equations of mass, momentum, energy, and species for laminar double-diffusive convection in the horizontal cavity can be expressed as follows:

$$
\begin{gathered}
\frac{\partial \rho}{\partial t}+\nabla \cdot \rho \boldsymbol{V}=0 \\
\rho\left(\frac{\partial \boldsymbol{V}}{\partial t}+\boldsymbol{V} \nabla \boldsymbol{V}\right)=\rho \boldsymbol{g}-\nabla p_{d y n}+\nabla \cdot \mu \nabla \boldsymbol{V}+\frac{1}{3} \nabla \mu \nabla \cdot \boldsymbol{V} \\
\rho c_{p}\left(\frac{\partial T}{\partial t}+\boldsymbol{V} \nabla T\right)=(\nabla \cdot k \nabla T)+\frac{d \bar{p}}{d t} \\
\rho\left(\frac{\partial C}{\partial t}+\boldsymbol{V} \nabla C\right)=(\nabla \cdot \rho D \nabla C)
\end{gathered}
$$

$V$ is the velocity vector of components $(u, v)$.

The system of conservation equations is completed by the ideal gas law used in order to determine the mixture density field $\rho$ for a binary mixture of gases with molecular weights $M_{1}$ and $M_{2}$,

$$
\bar{p}=\sum_{i=1}^{N} \bar{p}_{i}=\frac{n R T}{\Omega}=\frac{R T}{\Omega} \sum_{i=1}^{N} n_{i}=\frac{R T}{\Omega} \sum_{i=1}^{N} \frac{m_{i}}{M_{i}},
$$

$M_{i}$ : molecular weight of the ith component. $m_{i}$ : mass of component (i). $n_{i}$ : number of moles of component (i). With: $m=n \cdot M$ total mass and $\rho=\frac{m}{\Omega}$ mix density.

We therefore have

$$
\begin{aligned}
& \bar{p}=\frac{R T}{\Omega} \sum_{i=1}^{N} \frac{m_{i}}{M_{i}}=\frac{\rho R T}{m} \sum_{i=1}^{N} \frac{m_{i}}{M_{i}}, \\
& \bar{p}=\frac{R T}{\Omega} \sum_{i=1}^{N} \frac{m_{i}}{M_{i}}=\rho R T \sum_{i=1}^{N} \frac{\left(\frac{m_{i}}{m}\right)}{M_{i}}=\rho R T \sum_{i=1}^{N} \frac{C_{i}}{M_{i}},
\end{aligned}
$$

$C_{i}$ is the concentration, mass fraction.

Since we only have two constituents (air and an other gas), we will have:

$$
\begin{aligned}
\bar{p} & =\rho R T \sum_{i=1}^{N} \frac{C_{i}}{M_{i}}=\rho R T\left(\frac{C_{1}}{M_{1}}+\frac{C_{2}}{M_{2}}\right)=\rho \frac{R}{M_{1}} T\left(C_{1}+\frac{M_{1}}{M_{2}} C_{2}\right) \\
& =\rho \frac{R}{M_{1}} T\left(C_{1}+\frac{M_{1}}{M_{2}}\left(1-C_{1}\right)\right) .
\end{aligned}
$$

Therefore,

$$
\bar{p}=\rho R^{*} T\left(C_{1}\left(1-M^{*}\right)+M^{*}\right) .
$$

with

$$
R^{*}=\frac{R}{M_{1}} \text { and } M^{*}=\frac{M_{1}}{M_{2}} .
$$

It is also assumed that the dynamic viscosity is, depending on the case studied, taken either constant $\mu(T)=\mu\left(T_{0}\right)=\mu_{0}$ or given by the Sutherland law ${ }^{29}$

$$
\frac{\mu(T)}{\mu_{0}}=\left(\frac{T}{T_{0}}\right)^{3 / 2} \frac{T_{0}+S_{\mu}}{T+S_{\mu}},
$$

where $S_{\mu}=110.5 \mathrm{~K}, \mu_{0}=1.68 \times 10^{-5} \mathrm{~kg} \mathrm{~m}^{-1} \mathrm{~s}^{-1}$.

Thermal conductivity is given by

$$
k(T)=\mu(T) \frac{c_{p 0} k_{0}}{\nu_{0}} .
$$

More generally, the diffusion coefficient of gases into the air can be approximated by the following formula: ${ }^{31}$

$$
D=D_{0} \frac{p_{0}}{\bar{p}}\left(\frac{T}{T_{0}}\right)^{1.685} .
$$

The non-slip boundary conditions are imposed over the walls, so the boundary and initial conditions can be expressed as

$$
\begin{aligned}
& u=v=T=C_{1}=0 \text { at } t=0, \\
& u=v=0, T=T_{h}, C_{1}=C_{1 h} \text { for } 0 \leq y \leq H \text { and } x=0, \\
& u=v=0, T=T_{c}, C_{1}=C_{1 c} \text { for } 0 \leq y \leq H \text { and } x=H, \\
& u=v=0, \frac{\partial T}{\partial y}=0, \frac{\partial C_{1}}{\partial y}=0 \text { for } 0 \leq x \leq H \text { and } y=0, \\
& u=v=0, \frac{\partial T}{\partial y}=0, \frac{\partial C_{1}}{\partial y}=0 \text { for } 0 \leq x \leq H \text { and } y=H .
\end{aligned}
$$

\section{B. Additional equations}

In the numerical resolution of Eqs. (1)-(5), the problem that arises is that there are more unknowns than equations (at $2 \mathrm{D}$, there are seven unknowns). This comes from the decomposition of the pressure, which introduces the thermodynamic pressure $\bar{p}$ as an additional unknown. In order to close the problem, we need an additional equation representing the initial state, that of the conservation of the mass.

\section{Calculation of $\bar{p}$}

In an open cavity, the thermodynamic pressure is the ambient pressure

$$
\bar{p}(t)=p_{0}, \forall t
$$

In a closed cavity, it is determined by the conservation of the total mass

$$
\int_{\Omega} \rho d \Omega=M_{0}=\int_{\Omega} \rho_{0} d \Omega, \forall t .
$$

$\Omega$ is the volume of the system and $M_{0}$ is defined as the initial mass of the system. 
Using Eq. (7), we get

$\int_{\Omega} \frac{\bar{p}}{R^{*} T\left(C_{1}\left(1-M^{*}\right)+M^{*}\right)} d \Omega=\int_{\Omega} \frac{p_{0}}{R^{*} T_{0}\left(C_{10}\left(1-M^{*}\right)+M^{*}\right)} d \Omega$.

Therefore,

$$
\bar{p}=p_{0} \frac{\int_{\Omega} \frac{1}{T_{0}\left(M^{*}+C_{10}\left(1-M^{*}\right)\right)} d \Omega}{\int_{\Omega} \frac{1}{T\left(M^{*}+C_{1}\left(1-M^{*}\right)\right)} d \Omega} .
$$

\section{Calculation of $\frac{d \bar{P}}{d t}$}

The second additional equation is given by the calculation of the term $d \bar{p} / d t$. The mass conservation equation, associated with the energy equation and the equation of state, allows writing the divergence of the velocity. According to Eq. (7), we have

$$
\begin{aligned}
\frac{d \rho}{d t}=\frac{1}{R^{*} T\left(M^{*}+C_{1}\left(1-M^{*}\right)\right)} \frac{d \bar{p}}{d t} & -\frac{\bar{p}}{R^{*} T^{2}\left(M^{*}+C_{1}\left(1-M^{*}\right)\right)} \frac{d T}{d t} \\
& -\frac{\bar{p}\left(1-M^{*}\right)}{R^{*} T\left(M^{*}+C_{1}\left(1-M^{*}\right)\right)^{2}} \frac{d C_{1}}{d t} .
\end{aligned}
$$

From equations of continuity, energy, and concentration, we get

$$
\begin{gathered}
\nabla \cdot \boldsymbol{V}=-\frac{1}{\rho} \frac{d \rho}{d t}, \\
\frac{d T}{d t}=\frac{1}{\rho c_{p}} \nabla \cdot k \nabla T+\frac{1}{\rho c_{p}} \frac{d \bar{p}}{d t}, \\
\frac{d C_{1}}{d t}=\frac{1}{\rho} \nabla \cdot \rho D \nabla C_{1},
\end{gathered}
$$

with $c_{p}=\frac{\gamma R^{*}}{\gamma-1}$

So,

$$
\begin{aligned}
\nabla \cdot \boldsymbol{V} & =-\frac{1}{\rho R^{*} T\left(M^{*}+C_{1}\left(1-M^{*}\right)\right)} \frac{d \bar{p}}{d t}+\frac{\bar{p}}{\rho R^{*} T^{2}\left(M^{*}+C_{1}\left(1-M^{*}\right)\right)} \frac{d T}{d t} \\
& +\frac{\bar{p}\left(1-M^{*}\right)}{\rho R^{*} T\left(M^{*}+C_{1}\left(1-M^{*}\right)\right)^{2}} \frac{d C_{1}}{d t} .
\end{aligned}
$$

Taking into account the equation of state given by (7), Eq. (19) becomes then:

$$
\begin{aligned}
\nabla \cdot \boldsymbol{V}=\frac{1}{\bar{p}} & {\left[\left(\frac{\gamma-1}{\gamma}\left(M^{*}+C_{1}\left(1-M^{*}\right)\right)-1\right) \frac{d \bar{p}}{d t}+\frac{\gamma-1}{\gamma}\right.} \\
& \left.\times\left(M^{*}+C_{1}\left(1-M^{*}\right)\right) \nabla \cdot k \nabla T+R^{*} T\left(1-M^{*}\right) \nabla . \rho D \nabla C_{1}\right] .
\end{aligned}
$$

For impermeable wall conditions,

$$
\int_{\Omega^{*}} \nabla \cdot V d \Omega=\int_{S} \boldsymbol{V} \cdot \boldsymbol{n} \cdot d S=0 .
$$

So,

$$
\begin{aligned}
\int_{\Omega} \nabla \cdot V d \Omega= & \int_{\Omega} \frac{1}{\bar{p}}\left[\left(\frac{\gamma-1}{\gamma}\left(M^{*}+C_{1}\left(1-M^{*}\right)\right)-1\right) \frac{d \bar{p}}{d t}+\frac{\gamma-1}{\gamma}\right. \\
& \left.\times\left(M^{*}+C_{1}\left(1-M^{*}\right)\right) \nabla \cdot k \nabla T+R^{*} T\left(1-M^{*}\right) \nabla \cdot \rho D \nabla C_{1}\right],
\end{aligned}
$$

$\bar{p}$ depends only on time. The application of the divergence theorem gives

$$
\begin{aligned}
\frac{d \bar{p}}{d t}= & \frac{1}{\int_{\Omega} d \Omega-\int_{\Omega} \frac{\gamma-1}{\gamma}\left(M^{*}+C_{1}\left(1-M^{*}\right)\right) d \Omega} \\
& \times\left[\frac{\gamma-1}{\gamma}\left(M^{*}+C_{1}\left(1-M^{*}\right)\right) \int_{S} k \frac{\partial T}{\partial n} d S+\frac{c^{2}}{\gamma}\left(1-M^{*}\right)\right. \\
& \left.\times \int_{S} \rho D \frac{\partial C_{1}}{\partial n} d S\right],
\end{aligned}
$$

where the speed of sound in an ideal gas $c$ is given by: $c=\sqrt{\gamma R^{*} T}$.

\section{Dimensionless form of equations}

The following parameters are used to put the equations in dimensionless form:

$$
\begin{gathered}
\tau=\frac{\alpha t}{H^{2}}, X=\frac{x}{H}, Y=\frac{y}{H}, U=\frac{u H}{\alpha}, V=\frac{v H}{\alpha}, \\
\Pi=\left(p-\bar{p}+\rho_{0} g y\right) / \rho_{0}\left(\alpha_{0} / H\right)^{2}, \bar{P}=\frac{\bar{p}}{p_{0}}, \theta=\frac{T-T_{0}}{\Delta T}, \\
W=\frac{C_{1}-C_{10}}{\Delta C_{1}} \text { or } \Delta T=T_{h}-T_{c}, T_{0}=\frac{T_{h}+T_{c}}{2} \text { and } \\
\Delta C_{1}=C_{1 h}-C_{1 c}, C_{10}=\frac{C_{1 h}+C_{1 c}}{2} .
\end{gathered}
$$

The thermo-physical properties (density, dynamic viscosity, thermal conductivity, thermal diffusivity, and mass diffusivity) are scaled, respectively, by $\rho_{0}, \mu_{0}, k_{0}, \alpha_{0}, D_{0}$ where the subscript 0 denotes values at the reference temperature $T_{0}$. The influence of the temperature on the specific heat is assumed to be negligible so that $c_{p} / c_{p 0}=1$. $^{24}$

The system of equations is expressed in non-dimensional conservative form $^{27}$ as

$$
\frac{\partial \rho^{*}}{\partial \tau}+\frac{\partial \rho^{*} U}{\partial X}+\frac{\partial \rho^{*} V}{\partial Y}=0
$$

$$
\begin{aligned}
\rho^{*}\left(\frac{\partial U}{\partial \tau}\right. & \left.+U \frac{\partial U}{\partial X}+V \frac{\partial U}{\partial Y}\right)= \\
& -\frac{\partial \Pi}{\partial X}+\operatorname{Pr}\left\{\frac{\partial}{\partial X}\left(\mu^{*} \frac{\partial U}{\partial X}\right)+\frac{\partial}{\partial Y}\left(\mu^{*} \frac{\partial U}{\partial Y}\right)+\frac{1}{3} \nabla \mu^{*} \nabla \cdot V^{*}\right\},
\end{aligned}
$$

$$
\begin{gathered}
\rho^{*}\left(\frac{\partial V}{\partial \tau}+U \frac{\partial V}{\partial X}+V \frac{\partial V}{\partial Y}\right)=-\frac{\partial \Pi}{\partial Y}-R a \operatorname{Pr} \frac{\rho^{*}-1}{2 \varepsilon_{T}}+ \\
\operatorname{Pr}\left\{\frac{\partial}{\partial X}\left(\mu^{*} \frac{\partial V}{\partial X}\right)+\frac{\partial}{\partial Y}\left(\mu^{*} \frac{\partial V}{\partial Y}\right)+\frac{1}{3} \nabla \mu^{*} \nabla \cdot V^{*}\right\}
\end{gathered}
$$




$$
\begin{aligned}
\rho^{*}\left(\frac{\partial \theta}{\partial \tau}+U \frac{\partial \theta}{\partial X}+V \frac{\partial \theta}{\partial Y}\right)= & \frac{\partial}{\partial X}\left(k^{*} \frac{\partial \theta}{\partial X}\right)+\frac{\partial}{\partial Y}\left(k^{*} \frac{\partial \theta}{\partial Y}\right) \\
& +\frac{\gamma-1}{2 \varepsilon_{T} \gamma} \frac{d \bar{P}}{d \tau},
\end{aligned}
$$$$
\rho^{*}\left(\frac{\partial W}{\partial t}+U \frac{\partial W}{\partial X}+V \frac{\partial W}{\partial Y}\right)=\frac{1}{L e}\left(\frac{\partial}{\partial X}\left(\rho^{*} D^{*} \frac{\partial W}{\partial X}\right)\right.
$$$$
\left.+\frac{\partial}{\partial Y}\left(\rho^{*} D^{*} \frac{\partial W}{\partial Y}\right)\right)
$$

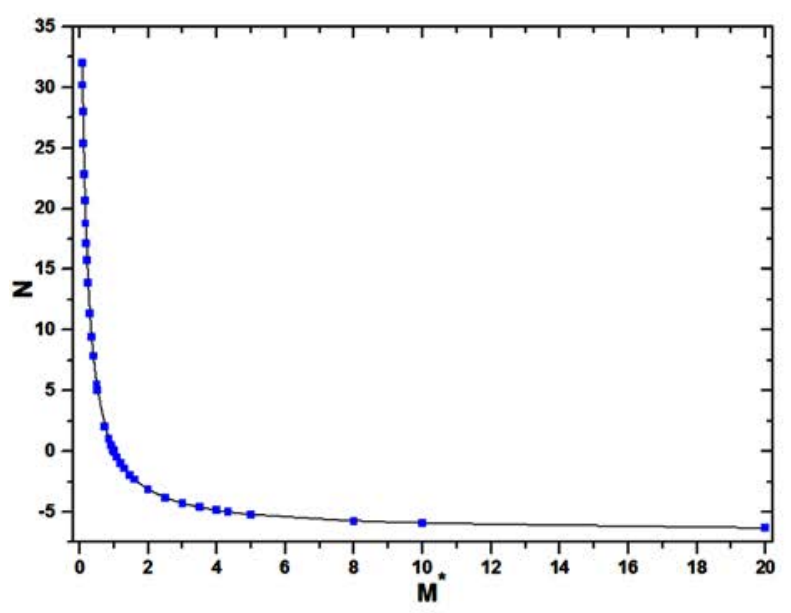

(a)

$$
\begin{gathered}
\bar{p}=\frac{\int_{\Omega^{2}} d \Omega^{*}}{\int_{\Omega^{*}} \frac{1}{\left(2 \varepsilon_{T} \theta+1\right)\left(1+\varepsilon_{m} W\right)} d \Omega^{*}}, \\
\rho^{*}=\frac{\bar{p}}{\left(1+2 \varepsilon_{T} \theta\right)\left(1+\varepsilon_{m} W\right)}, \\
\frac{d \bar{P}}{d \tau}=\frac{1}{\int_{\Omega^{*}} \frac{d \Omega^{*}}{M^{*}+C_{10}\left(1-M^{*}\right)}-\int_{\Omega^{*}} \frac{\gamma-1}{\gamma}\left(1+\varepsilon_{m} W\right) d \Omega^{*}} \\
\times\left[2 \varepsilon_{T}\left(1+\varepsilon_{m} W\right) \int_{S^{*}} k^{*} \frac{\partial \theta}{\partial n} d S^{*}+\frac{\varepsilon_{m}}{L e} \int_{S^{*}} \rho^{*} D^{*} \frac{\partial W}{\partial n} d S^{*}\right],
\end{gathered}
$$

\begin{tabular}{|c|c|c|c|c|}
\hline & Heuveline $^{33}$ & Vierendeels $s^{34}$ & \multicolumn{2}{|c|}{ Present work } \\
\hline \multicolumn{5}{|c|}{ Test case $1\left(\mathrm{Ra}=10^{6}, \varepsilon_{b}=0.6\right.$, and constant properties) } \\
\hline $\mathrm{Nu}(\mathrm{h})$ & 8.859778 & 8.85978 & 8.85847 & \\
\hline $\mathrm{Nu}(\mathrm{c})$ & 8.85978 & 8.85978 & 8.85847 & \\
\hline $\bar{p} / p_{0}$ & 0.85634 & 0.856340 & 0.85584 & \\
\hline \multicolumn{5}{|c|}{ Test case $2\left(\mathrm{Ra}=10^{6}, \varepsilon_{b}=0.6\right.$, and Sutherland law $)$} \\
\hline $\mathrm{Nu}(\mathrm{h})$ & 8.6889 & 8.6866 & 8.70078 & 8.68451 \\
\hline $\mathrm{Nu}(\mathrm{c})$ & 8.6831 & 8.6866 & 8.70057 & 8.68316 \\
\hline $\bar{p} / p_{0}$ & 0.9249 & 0.924489 & 0.92498 & 0.92425 \\
\hline Type of Mesh & 400000 & $2048 \times 2048$ & $300 \times 300$ & $202 \times 202$ \\
\hline
\end{tabular}

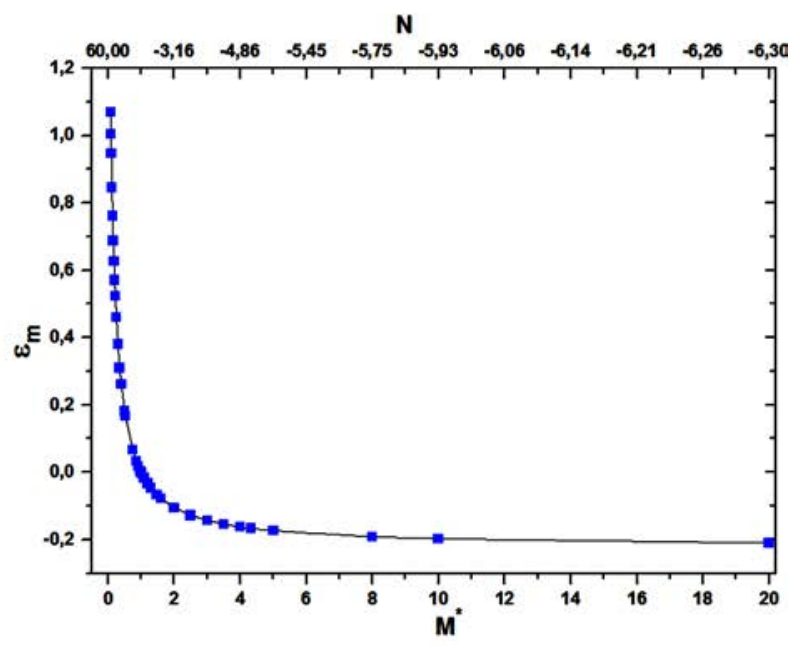

(b)

FIG. 2. Evolution of (a) $N$ and (b) $\varepsilon_{m}$ as a function of $M^{*}(N)$.

TABLE I. Air benchmark test cases. ${ }^{33,34}$

TABLE II. Buoyancy ratio parameter as a function of $M^{*}$ and $\varepsilon_{m}$

\begin{tabular}{lcccccccccccc}
\hline$N$ & 5 & 2 & 1 & 0.5 & 0.1 & 0 & -0.1 & -0.5 & -1 & -2 & -5 \\
\hline$M^{*}$ & 0.524 & 0.744 & 0.855 & 0.922 & 0.984 & 1 & 1.017 & 1.09 & 1.196 & 1.476 & 4.333 \\
$\varepsilon_{m}$ & 0.167 & 0.0667 & 0.0333 & 0.0167 & 0.00333 & 0 & -0.0033 & -0.0167 & -0.0333 & -0.0667 & -0.167 \\
\hline
\end{tabular}


TABLE III. Average Nusselt and Sherwood numbers along the hot wall, $L e=1, \operatorname{Pr}=0.71$, and $R a=10^{7}$.

\begin{tabular}{|c|c|c|c|c|c|c|c|c|c|}
\hline$M^{*}$ & 1.00167 & 1.017 & 1.0344 & 1.09 & 1.1515 & 1.1734 & 1.196 & 1.3226 & 4.3333 \\
\hline$\varepsilon_{m}$ & -0.0003 & -0.0033 & -0.0067 & -0.0167 & 0.0267 & -0.03 & -0.033 & -0.05 & -0.167 \\
\hline$N$ & -0.01 & -0.1 & -0.2 & -0.5 & -0.8 & -0.9 & -1 & -1.5 & -5.0 \\
\hline \multirow{2}{*}{$\begin{array}{l}N u(S h) \text { present study } \\
35\end{array}$} & 16.465 & 16.049 & 15.55 & 13.69 & 10.67 & 8.81 & \multirow[t]{4}{*}{2.88} & 13.69 & 23.86 \\
\hline & 16.4 & 16.0 & 15.5 & 13.6 & 10.6 & 8.8 & & 13.6 & 23.7 \\
\hline 20 & 16.3 & 15.9 & 15.4 & 13.5 & 10.5 & 8.6 & & 13.5 & 13.6 \\
\hline 36 & 16.7 & 16.0 & 15.3 & 13.6 & 10.6 & 8.8 & & 13.5 & 23.7 \\
\hline
\end{tabular}

where $\varepsilon_{m}=\frac{\Delta C_{1}\left(1-M^{*}\right)}{M^{*}+C_{10}\left(1-M^{*}\right)}$ and $\varepsilon_{T}=\frac{\Delta T}{2 T_{0}}$.

Note that

$$
\begin{aligned}
& M^{*} \rightarrow 0, \varepsilon_{m} \\
& M^{*} \rightarrow \frac{\Delta C_{1}}{C_{10}}, \\
& \rightarrow \infty, \varepsilon_{m} \rightarrow \frac{\Delta C_{1}}{C_{10}-1} .
\end{aligned}
$$

with $C_{10}=\frac{\Delta C_{1}}{2}$
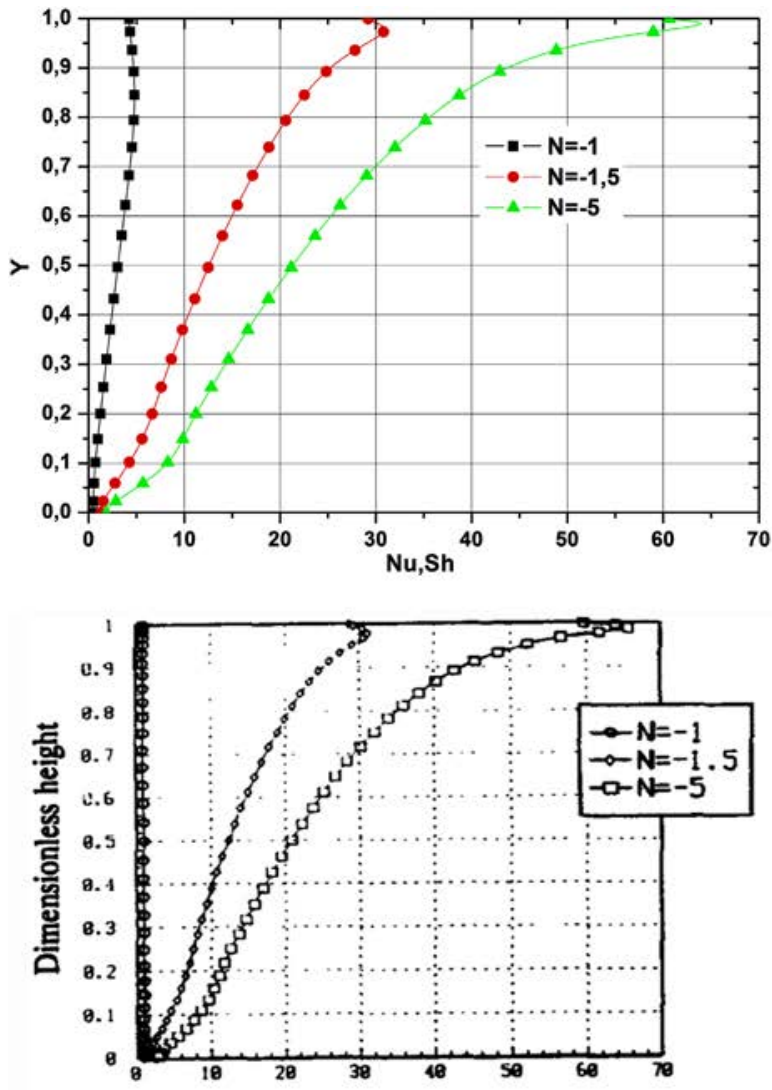

Nusselt mumber along the hot wall

$$
\begin{gathered}
M^{*} \rightarrow 0, \varepsilon_{m} \rightarrow 2, \\
M^{*} \rightarrow \infty, \varepsilon_{m} \rightarrow \frac{\Delta C_{1}}{\frac{\Delta C_{1}}{2}-1} .
\end{gathered}
$$

The corresponding boundary conditions are

$U=V=0, \theta=\theta_{c}=0.5, W=W_{c}=0.5$ at $X=0,0 \leq Y \leq 1$,

$U=V=0, \theta=\theta_{h}=-0.5, W=W_{h}=-0.5$ at $X=1,0 \leq Y \leq 1$,
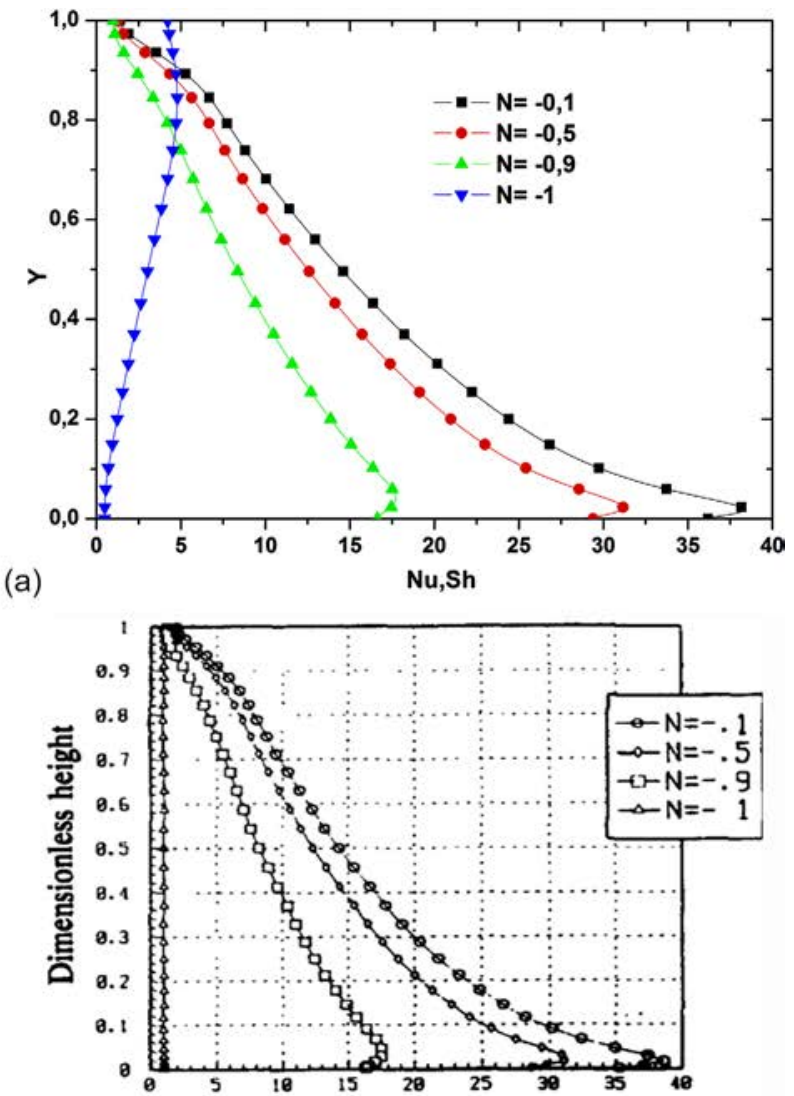

(b)

FIG. 3. Distributions of local Nusselt numbers on the hot wall in (a) present study and (b) ${ }^{35}$ at $R a=10^{7}$ and $L e=1$. 
$U=V=0,-\frac{\partial \theta}{\partial Y}=0,-\frac{\partial W}{\partial Y}=0$ at $Y=0,0 \leq X \leq 1$,

$U=V=0, \frac{\partial \theta}{\partial Y}=0, \frac{\partial W}{\partial Y}=0$, at $Y=1,0 \leq X \leq 1$. by

The transport coefficients $\mu^{*}(\theta)$ and $k^{*}(\theta)$ are given, respectively,

$$
\begin{gathered}
\mu^{*}=\left(2 \varepsilon_{T} \theta+1\right)^{3 / 2} \frac{\left(1+S_{\mu} / T_{0}\right)}{2 \varepsilon_{T} \theta+1+S_{\mu} / T_{0}}, \\
k^{*}=\frac{\mu^{*}}{\operatorname{Pr}} .
\end{gathered}
$$

The mass diffusion coefficient is given by

$$
D^{*}=\frac{1}{\bar{P}}\left(2 \varepsilon_{T} \theta+1\right)^{1.685}
$$

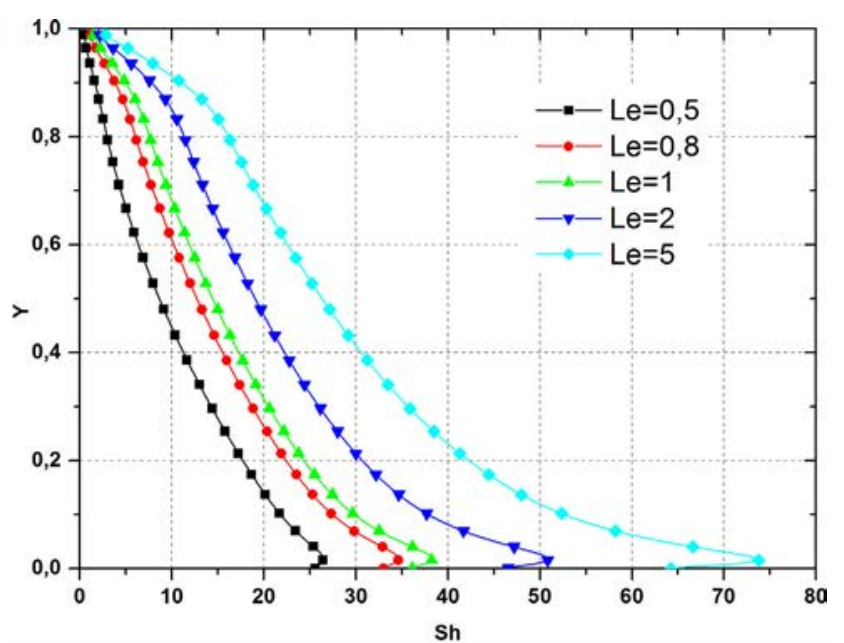

(a)

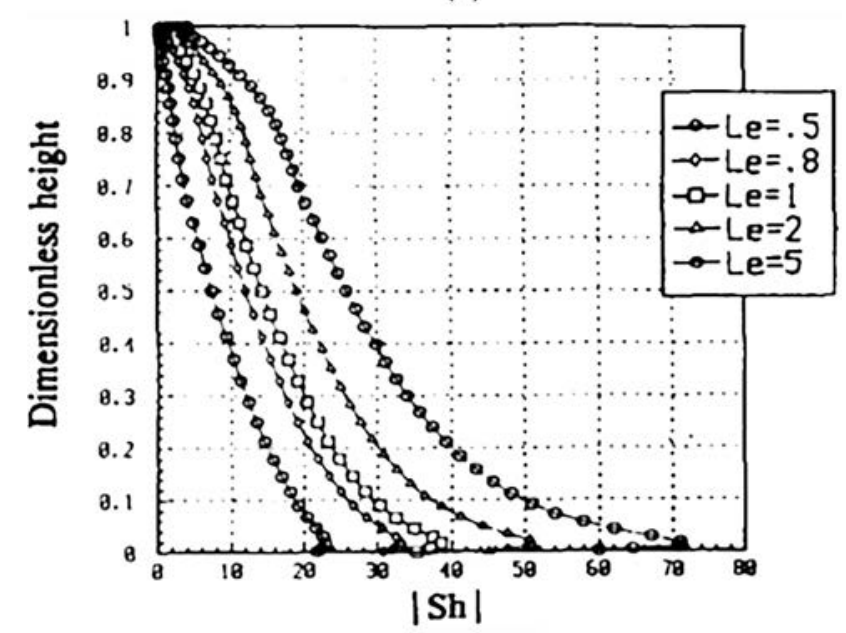

(b)

FIG. 4. Distributions of local Sherwood numbers on the hot wall in (a) present study and $(b)^{35}$ at $R a=10^{7}$ and $N=-0.1$.
To characterize heat and mass transfer of the double-diffusive convection in the cavity, the local Nusselt number $\mathrm{Nu}$ and Sherwood number $S h$ on the heated vertical surface are defined by

$$
\begin{gathered}
N u=-\left.\frac{\partial \theta}{\partial X}\right|_{X=0}, \\
S h=-\left.\rho^{*} D^{*} \frac{\partial W}{\partial X}\right|_{X=0} .
\end{gathered}
$$

The average convective Nusselt number and Sherwood number are calculated by integrating the temperature and concentration gradient over the vertical wall as

$$
\begin{aligned}
N u_{c_{\text {avg }}} & =\int_{0}^{1} N u(X) d X \\
S h_{\text {avg }} & =\int_{0}^{1} \operatorname{Sh}(X) d X
\end{aligned}
$$

\section{Boussinesq approximation recovery}

Boussinesq's approximation is characterized by a small difference in temperature and concentration $\left(\varepsilon_{T} \rightarrow 0, \varepsilon_{m} \rightarrow 0\right)$, a zero velocity divergence $(\nabla \cdot \vec{V}=0)$, and thermodynamic pressure tending toward unity $(\bar{P}=1)$.

If we hold these hypotheses, Eq. (39) becomes

$$
\rho^{*}=\frac{1}{\left(1+2 \varepsilon_{T} \theta\right)\left(1+\varepsilon_{m} W\right)} \mid \begin{aligned}
& \varepsilon_{T} \rightarrow 0 \\
& \varepsilon_{m} \rightarrow 0
\end{aligned} .
$$

The term $\frac{\rho^{*}-1}{2 \varepsilon_{T}}$ in Eq. (35) becomes

$$
\frac{\rho^{*}-1}{2 \varepsilon_{T}}=-\frac{\varepsilon_{m} W}{2 \varepsilon_{T}}-\theta-\varepsilon_{m} \theta W .
$$

So, for $\left(\varepsilon_{T} \rightarrow 0, \varepsilon_{m} \rightarrow 0\right)$

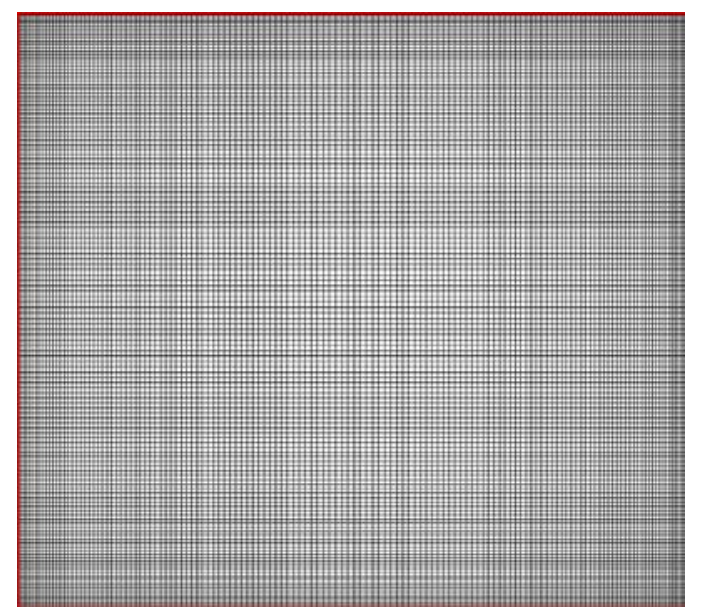

FIG. 5. Near-wall refinement for grid using exponential distribution. 


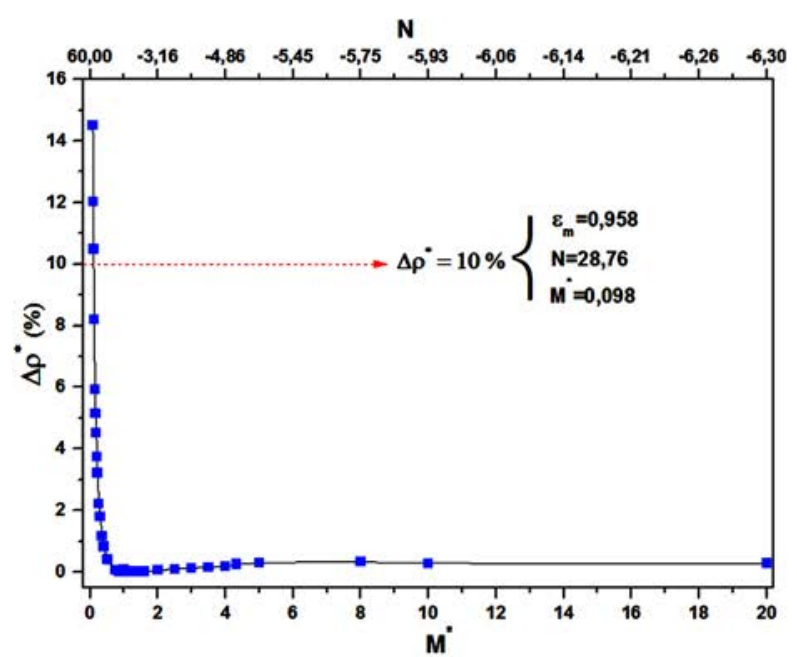

(a)

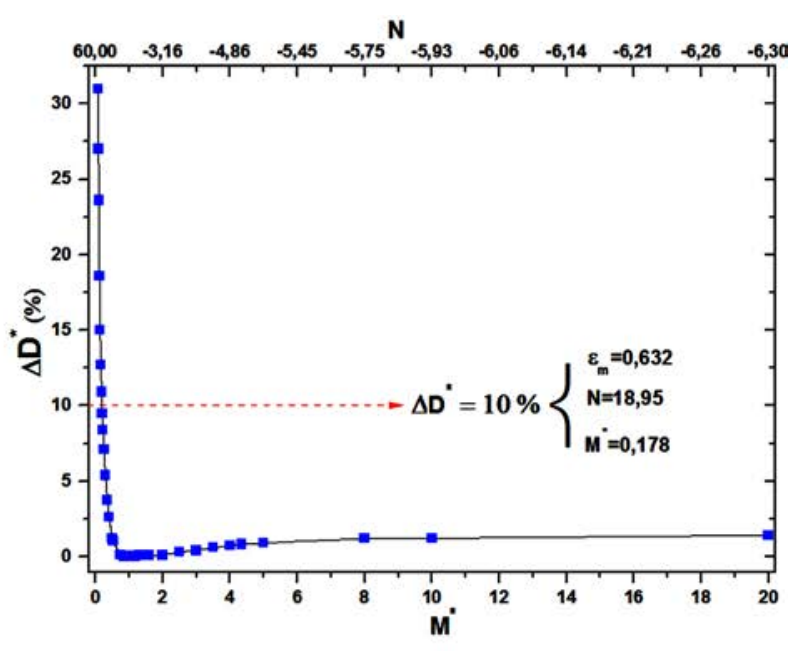

(b)

FIG. 6. Rate of change of (a) $\rho^{*}$ and (b) $D^{*}$ as a function of $M^{*}(\mathrm{~N})$ at $R a=10^{6}$ and $L e=1$.

$$
\frac{\rho^{*}-1}{2 \varepsilon_{T}} \rightarrow-\frac{\varepsilon_{m} W}{2 \varepsilon_{T}}-\theta,
$$

where $\frac{\varepsilon_{m}}{2 \varepsilon_{T}}$ tend toward a value $N$ (positive or negative, depending on the sign of $\left.\varepsilon_{m}\right)$ and which represents buoyancy ratio $N=\frac{\beta_{s} \Delta C_{1}}{\beta_{t} \Delta T}=\frac{\varepsilon_{m}}{2 \varepsilon_{T}}$, and where

$$
\beta_{s}=\frac{\left(1-M^{*}\right)}{M^{*}+C_{10}\left(1-M^{*}\right)} \text { and } \beta_{t}=\frac{1}{T_{0}} .
$$

We get

$$
\begin{gathered}
\frac{\partial U}{\partial X}+\frac{\partial V}{\partial Y}=0 \\
\frac{\partial U}{\partial \tau}+U \frac{\partial U}{\partial X}+V \frac{\partial U}{\partial Y}=-\frac{\partial P}{\partial X}+\operatorname{Pr} \nabla U \\
\frac{\partial V}{\partial \tau}+U \frac{\partial V}{\partial X}+V \frac{\partial V}{\partial Y}=-\frac{\partial P}{\partial Y}+\operatorname{Pr} \nabla V+R a \operatorname{Pr}(\theta+N W), \\
\frac{\partial \theta}{\partial \tau}+U \frac{\partial \theta}{\partial X}+V \frac{\partial \theta}{\partial Y}=\nabla \theta \\
\frac{\partial W}{\partial t}+U \frac{\partial W}{\partial X}+V \frac{\partial W}{\partial Y}=\frac{1}{L e} \Delta W .
\end{gathered}
$$

\section{NUMERICAL SIMULATION}

The numerical solution of the governing differential equations for the velocity, pressure, and temperature fields is obtained by using a finite volume technique. A power scheme was also used in approximating advection-diffusion terms. The SIMPLER algorithm whose details can be found in Patankar ${ }^{32}$ with a staggered grid is employed to solve the coupling between pressure and velocity. The discretization equations were solved by the Gauss-Seidel method. The iteration method used in this program is a line-by-line procedure, which is a combination of the direct method and the resulting Tri Diagonal Matrix Algorithm (TDMA).

\section{VALIDATION}

In order to check on the accuracy of the numerical technique employed for the solution of the problem considered in the present study, we have first validated our numerical code to ensure that it delivers correct results. The code has been verified by comparing our results with those available in the literature.

Results for the problem of the pure natural convection for a large temperature gradients and for the problem of the double-diffusive convection for a low temperature and solutal concentration gradients are compared with those obtained by ${ }^{33,34}$ and, respectively. For the case of thermal convection for large

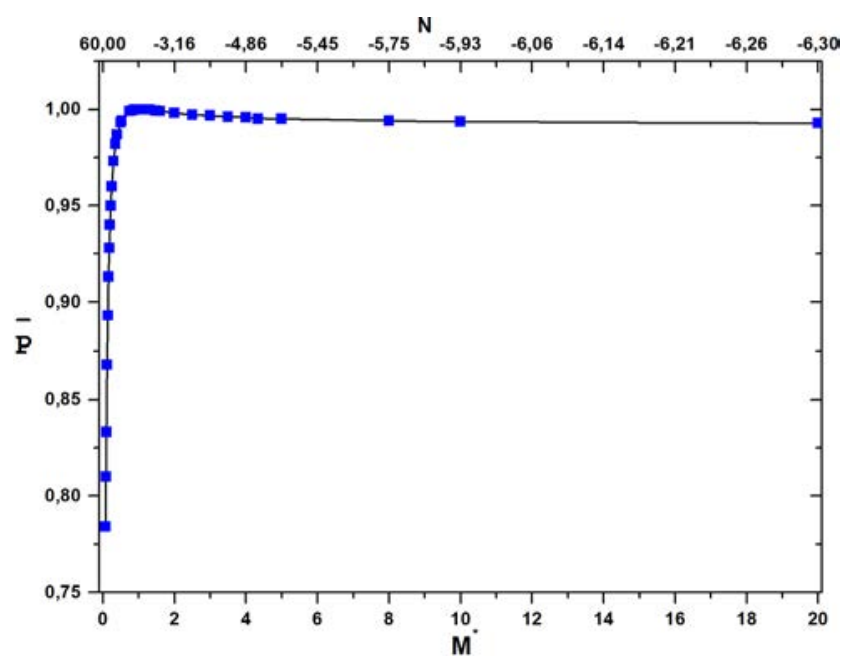

FIG. 7. Variation of the thermodynamic pressure $\bar{P}$ as a function of $M^{*}(\mathrm{~N})$ at $R a=10^{6}$ and $L e=1$. 


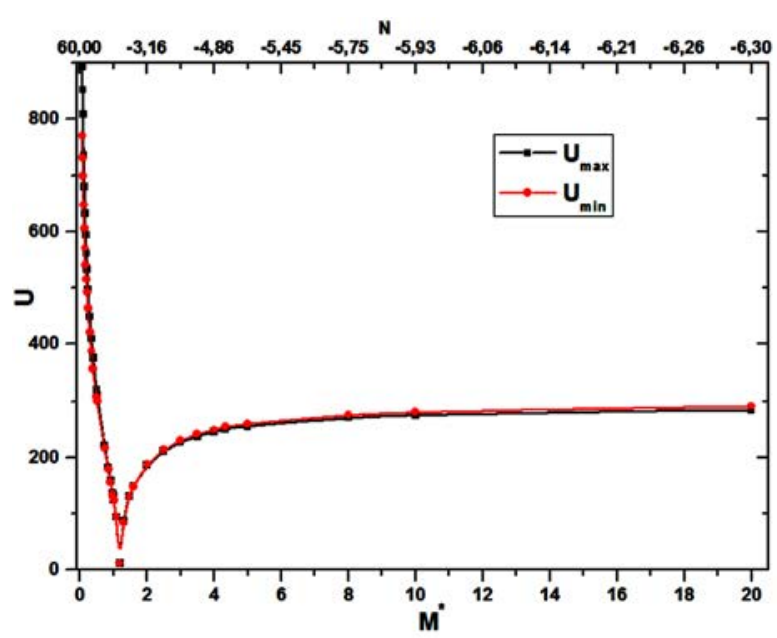

(a)

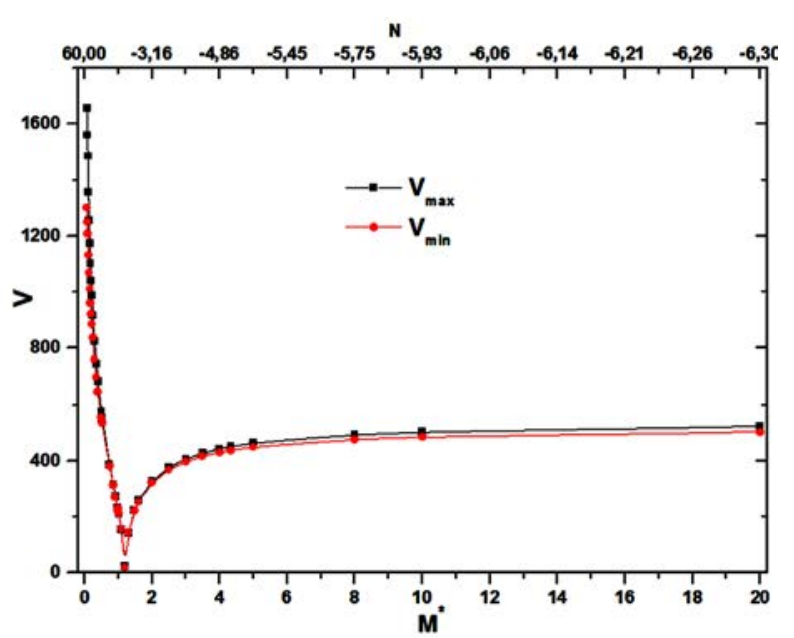

(b)

FIG. 8. Maximum and minimum (a) horizontal and (b) vertical velocities as a function of $M^{*}(N)$ at $R a=10^{6}$ and $L e=1$.

temperature gradients, calculations were carried out for air initially at temperature $T_{0}=600 \mathrm{~K}$ and for $R a=10^{6}$ and $\varepsilon_{T}=0.6$. The test cases include problems with constant and variable properties. Data presented in Table I, including average Nusselt numbers at the active walls and the pressure ratio $\bar{p} / p_{0}$, show a good agreement with results of Heuveline ${ }^{33}$ and Vierendeels. ${ }^{3}$

For double-diffusive convection, we used specified values of the molecular weight ratio $M^{*}$, which correspond to values of buoyancy ratio $N$ widely used in the literature. We also used weak thermal and solutal gradients to obtain quantitative bounds for the validity of the Boussinesq approximation.

Table II shows the values of buoyancy ratio $N=\frac{\varepsilon_{m}}{2 \varepsilon_{T}}$ corresponding to the different values of molecular weight ratio $M^{*}$ and nonBoussinesq solutal parameter $\varepsilon_{m}$.
The quantities $N$ and $\varepsilon_{m}$ are inversely proportional to $M^{*}$ and following a hyperbolic trajectory (Fig. 2), so that

$$
\begin{gathered}
M^{*} \rightarrow 0, \varepsilon_{m} \rightarrow \frac{\Delta C}{C_{0}}=2 \text { or; } N=\frac{\varepsilon_{m}}{2 \varepsilon_{T}}=60, \\
M^{*} \rightarrow \infty, \varepsilon_{m} \rightarrow \frac{\Delta C}{C_{0}-1}=-0.222 \text { or } ; N=\frac{\varepsilon_{m}}{2 \varepsilon_{T}}=-6.66 .
\end{gathered}
$$

Table III shows the values of average Nusselt and Sherwood numbers along the hot wall for $L e=1, \operatorname{Pr}=0.71$, and $R a=10^{7}$. Figures 3 and 4 show the distributions of the local Nusselt $(\mathrm{Nu})$ and Sherwood (Sh) numbers on the hot wall for over a range for buoyancy ratio $N$ and for $L e=1, \operatorname{Pr}=0.71$, and $R a=10^{7}$.

It can be seen from the comparison that the solution found by the low Mach number approximation approaches the Boussinesq

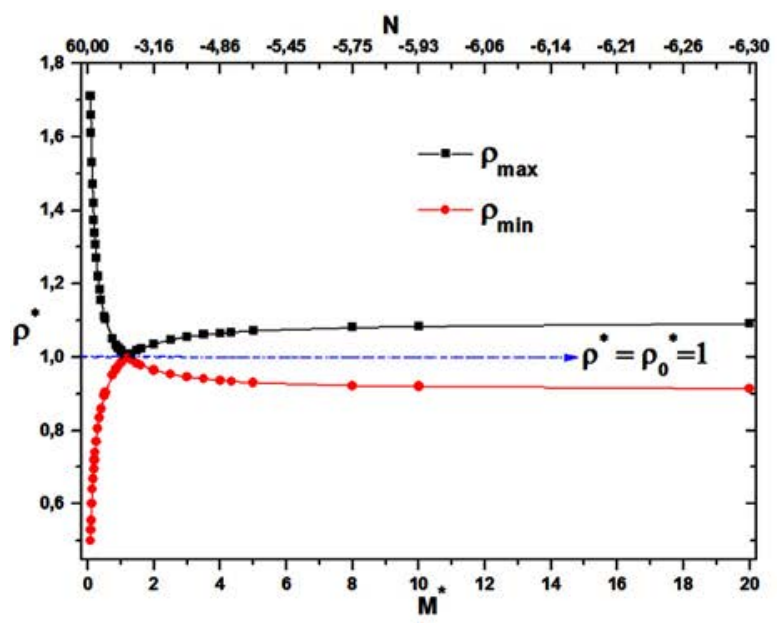

(a)

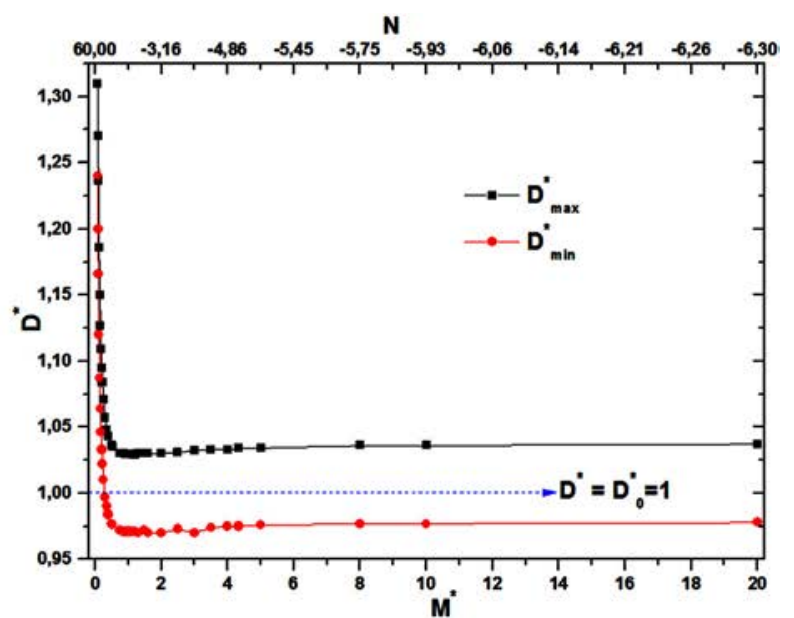

(b)

FIG. 9. Variation of the maximum and minimum (a) density and (b) mass diffusivity as a function of $M^{*}(N)$ at $R a=10^{6}$ and $L e=1$. 
solution, the present work is in a very good agreement with the previous works. Note that for the case $\mathrm{N}=-1\left(\mathrm{M}^{\star}=1.196\right)$ a slight deviation between the results is observed, which means that the nonBoussinesq effect begins to affect the flow. In this special case, the thermal and solutal buoyancy forces are canceled. Thereby, the convection motion disappears, and the heat and mass transfers are induced only by diffusion.

\section{RESULTS AND DISCUSSION}

This section is devoted to find a limit value for the buoyancy ratio $N$ in the Boussinesq approximation for natural convection flows evolving inside a close and differentially heated cavity, which is filled with an air-gas mixture, both for assisting and opposing flows depending on the molecular weight ratio parameter $M^{*}$.

The grid independence test is carried out to examine the dependence of numerical accuracy on grid sizes. The distribution of grids in the near walls was done using an exponential bunching methodology chosen in such a way that there is a greater mesh density near the wall as shown in Fig. 5. It turns out that the solution becomes independent of grid size at $202 \times 202$; this is a best trade-off between the accuracy and the computation time

Therefore, Prandtl (Pr), Rayleigh (Ra), Lewis $(L e)$ numbers, nonBoussinesq thermal parameter $\varepsilon_{T}$, and concentration gradient $(\Delta C)$ are fixed to $\operatorname{Pr}=0.71, R a=10^{6}, L e=1, \varepsilon_{T}=0.017(\Delta T=10 \mathrm{~K})$, and $\Delta C=0.2\left(20 \%, C_{0}=0.1\right)$, respectively. The controlled variables are the molecular weight ratio $0 \leq M^{*} \leq 20$, which corresponds to non-Boussinesq solutal parameter $\varepsilon_{m},-0.21 \leq \varepsilon_{m} \leq 1.07$ and to buoyancy ratio $N,-6.3 \leq N \leq 32$. The thermophysical properties are estimated at a reference temperature of $300 \mathrm{~K}$.

It should be noted that for Boussinesq conditions, the solutions remain symmetric, and consequently we always have the following results: $\rho_{\max }^{*} \approx \rho_{\min }^{*}, D_{\max }^{*} \approx D_{\min }^{*}, U_{\max } \approx U_{\min }, \quad V_{\max } \approx V_{\min }$, $\bar{P} \approx 1, N u \approx S h$ for $L e=1$, and also isotherms merge with isoconcentrations.

Depending on the molecular weight ratio, $M^{*}\left(M^{*} \succ 1\right.$, $\left.M^{*} \prec 1\right)$, the definition of $\varepsilon_{m}$ implies negative values of $\varepsilon_{m}$ when the molecular weight of the gas which diffuses into the cavity is higher than that of the inert gas $\left(M^{*} \succ 1\right.$, opposing flows) and vice versa ( $M^{*} \prec 1$, aiding flows). The solutal body force can either augment or oppose to the thermal body force.

The value $L e=1$ was used for the Lewis number to yield a similar distribution of the Nusselt and Sherwood numbers, as well as for the distribution of isovalues of temperature and concentration, this is only true in the Boussinesq approximation.

It is considered that the Boussinesq approximation is valid as long as the temperature/solutal difference in the fluid does not modify the physical properties by more than $10 \%$ compared to their values at the reference temperature $T_{0 .}{ }^{37}$

The results are presented in terms of streamlines, isotherms, local and average Nusselt and Sherwood numbers, and cross section of temperature and velocity profiles. To check the symmetry of the solution when the Boussinesq approximation is achieved, the results are also presented, as a function of molecular weight ratio $M^{*}$, in terms of rates of change of density $\Delta \rho^{*}$ and mass diffusivity $\Delta D^{*}$, maximum and minimum horizontal $\left(U_{\max }, U_{\min }\right)$ and vertical $\left(V_{\max }, V_{\min }\right)$ velocities, maximum and minimum density $\rho^{*}$ and mass diffusivity $D^{*}$, and thermodynamic pressure $\bar{P}$.

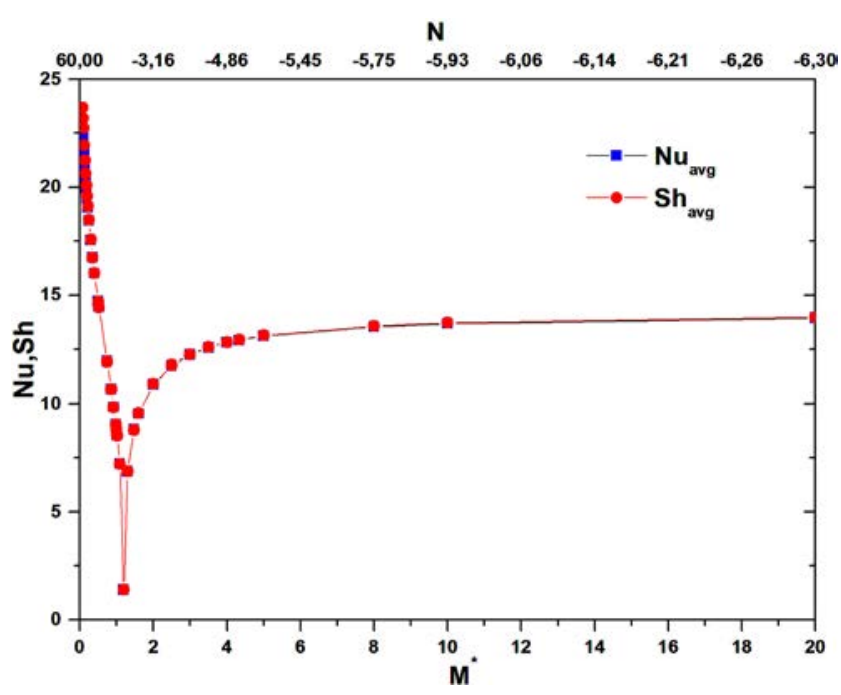

FIG. 10. Variation of the average Nusselt and Sherwood numbers at the heated surface as a function of $M^{*}(N)$ at $R a=10^{6}$ and $L e=1$.

The deviation from the Boussinesq approximation is investigated according to the value of $M^{*}$ in the range $0 \leq M^{*} \leq 20(-6.3 \leq N \leq 32)$ for a mass fraction at the walls $\Delta \bar{C}=20 \%$. Whatever their values, the thermal conductivity and kinematic viscosity always remain constant and equal to their values given by Sutherland's law. The thermophysical properties which change by changing the parameter $M^{*}$ are the density and the mass diffusivity.

According to Figs. 6(a) and 6(b), it can be seen that the effect of $M^{*}$ on the variation in mass diffusivity is greater than that on density.

Figures 6(a) and 6(b) show the evolution of the change rate in percentage of the density and of the mass diffusivity compared to their values in the reference state. It can be seen that the effect of $M^{*}$ on the variation in mass diffusivity is greater than that on density. The results show that for values of $M^{*} \leq 0.105(N \geq 27)$, the rate of variation of the density exceeds $10 \%$, while the rate of change of mass diffusivity

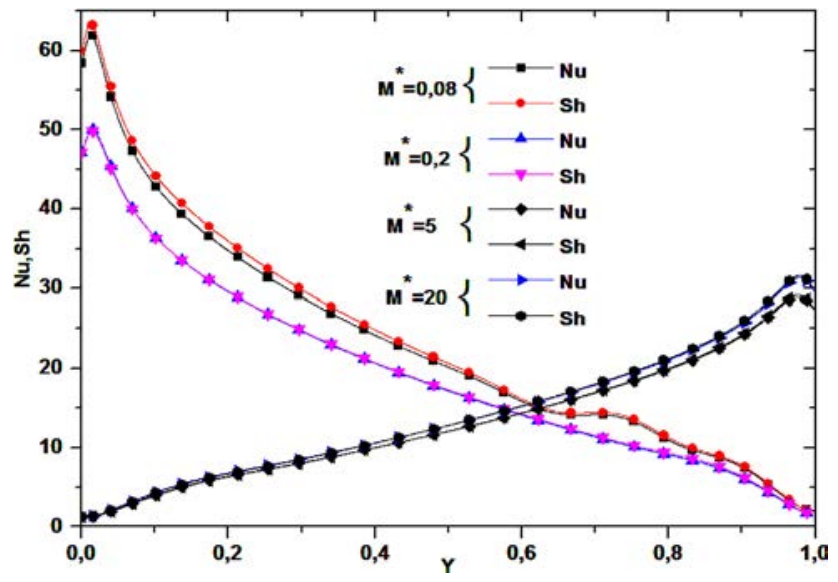

FIG. 11. Local Nusselt and Sherwood numbers on the hot wall $M^{*}(N)$ at $R a=10^{6}$ and $L e=1$. 

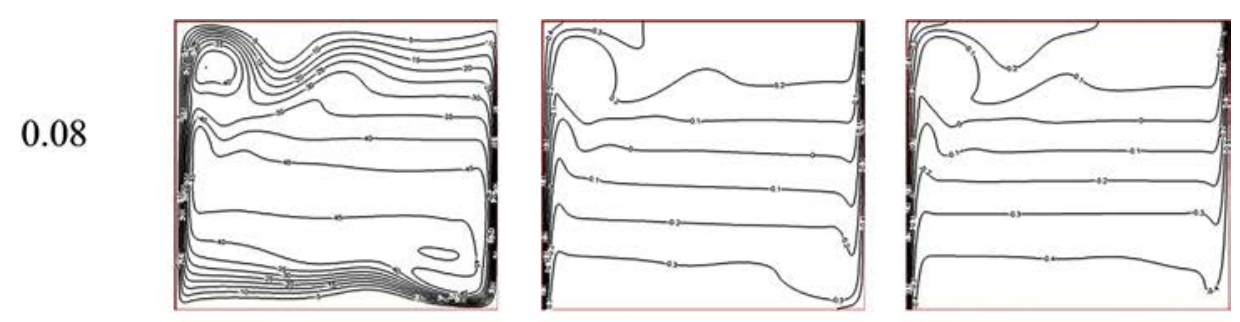

0.2
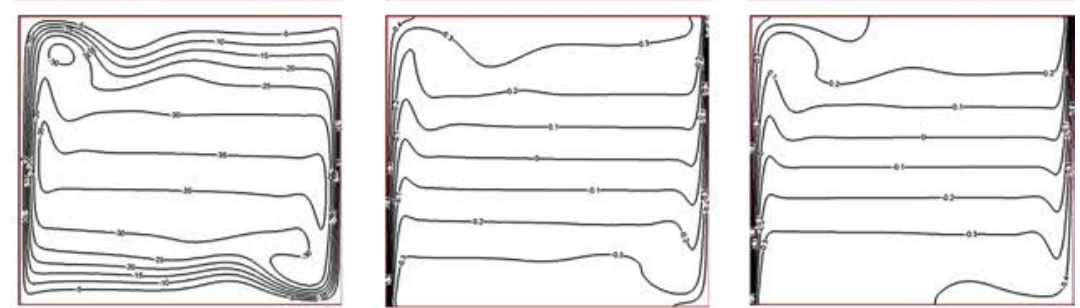

1
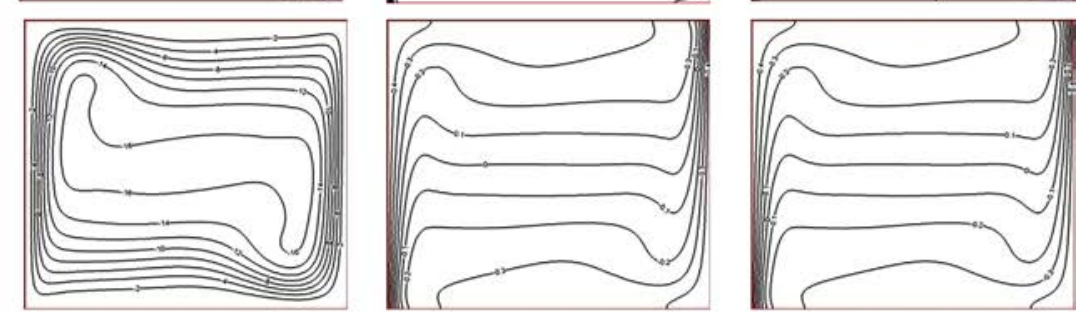

1.196
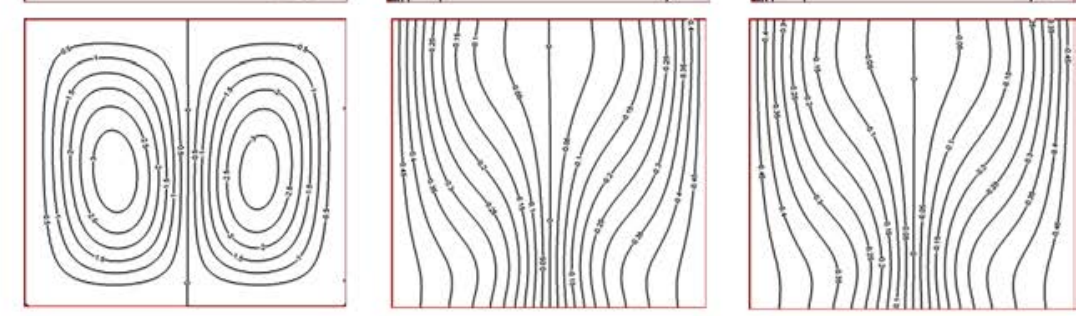

4.33
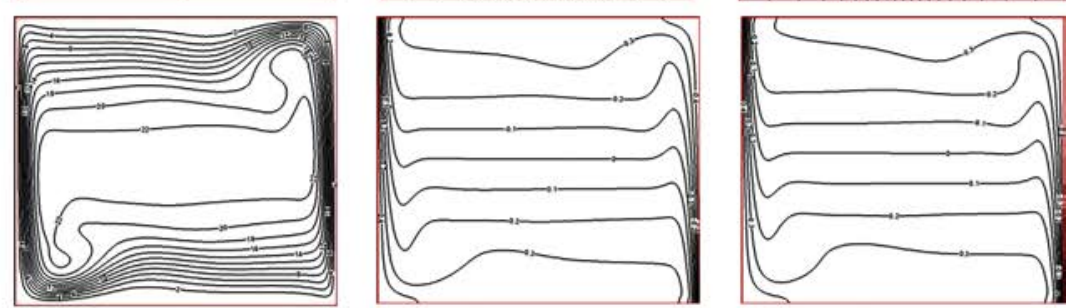

20
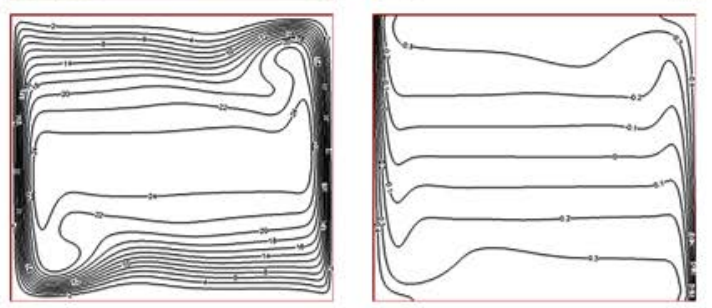

(b)

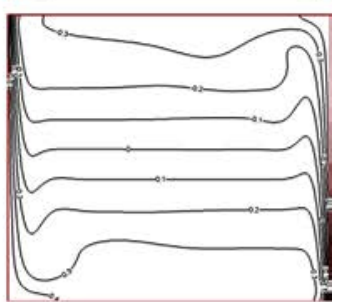

(c)

FIG. 12. (a) Streamlines, (b) temperature, and (c) concentration contours as a function of $M^{*}(N)$ at $R a=10^{6}$ and $L e=1$. 
exceeds $10 \%$ for values of $M^{*} \leq 0.178(N \geq 18)$. For this range of variation of $M^{*}$, Fig. 7 shows a significant decrease in the thermodynamic pressure, varying up to $17 \%$ from its reference value due to a loss of a large number of molecules between the initial and steady-state, while it is almost constant and tends toward unity if $M^{*} \geq 0.5$. This indicates that in the context of the Boussinesq approximation, the ratio $M^{*}$ must not be less than 0.105 corresponding to $N=27$. For values of $M^{*}$ less than 0.105 (i.e., for very light gases) compressibility has a considerable effect on the flow and the Boussinesq approximation is no longer valid and the low Mach number model must be used to account for the effects of compressibility.

For $M^{*} \leq 0.105$, a small decrease in $M^{*}$ leads to a large increase in velocities (Fig. 8) and a large decrease in thermodynamic pressure because the number of molecules within the cavity becomes much small. On the other hand, the pressure does not vary much for $M^{*} \geq$ $0.35(N \leq 9.4)$. A very important variation is also observed on the density and the mass diffusivity [Figs. 9(a) and 9(b)], compared to their reference values.

The local and average Nusselt and Sherwood numbers (Figs. 10 and 11) do not present a large deviation [2.66\% for $M^{*}=0.08$ $(\mathrm{N}=32)]$.

When the Boussinesq approximation is invoked, the temperature [Fig. 12(b)] and concentration [Fig. 12(c)] fields merge, it is difficult to delineate between isotherms and isoconcentration, the energy and mass fluxes at the walls are the same (Fig. 10). This symmetry is also found on the streamlines [Fig. 12(a)] plotted for very weak compressible flows. The deviation of the thermodynamic pressure from its value in the Boussinesq approximation $(\bar{P}=1)$ is small (Fig. 7$)$.

For values of $M^{*}$ close to 1 , results show that the steady-state $\mathrm{Nu}$ does not differ much from the Boussinesq value $(N u=8.82$, for the case of pure natural convection). ${ }^{27}$ The variation in density [Figs. 6(a) and 9(a)] and mass diffusivity [Figs. 6(b) and 9(b)] with respect to their reference values is negligible. The predictions of incompressible and compressible models did not differ significantly, and the solutions remain symmetric. These results are clearly seen in Figs. 12-15 for isocontours of streamlines, temperature and concentration, temperature at mid-height cavity, vertical velocity at the horizontal cross section,

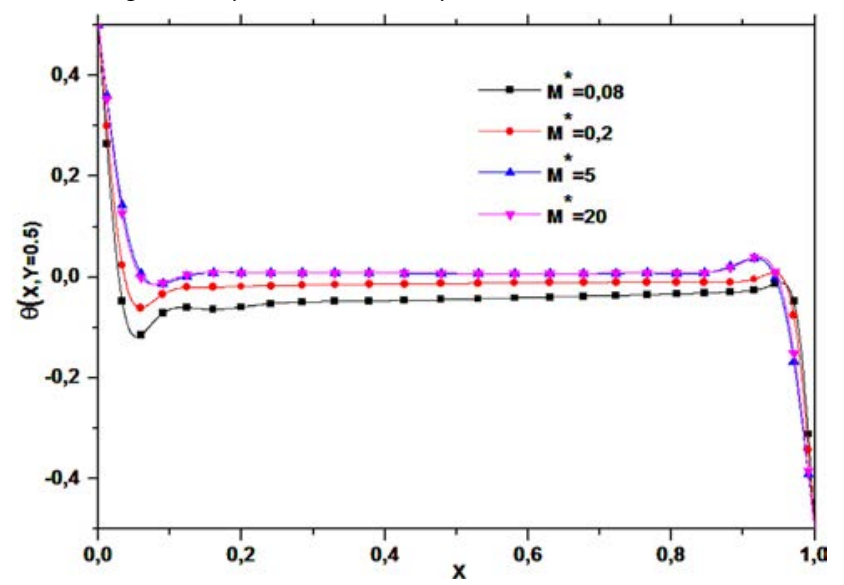

FIG. 13. Cross section of the temperature at mid-height cavity at $Y=0.5$ for $R a=$ $10^{6}$ and $L e=1$.

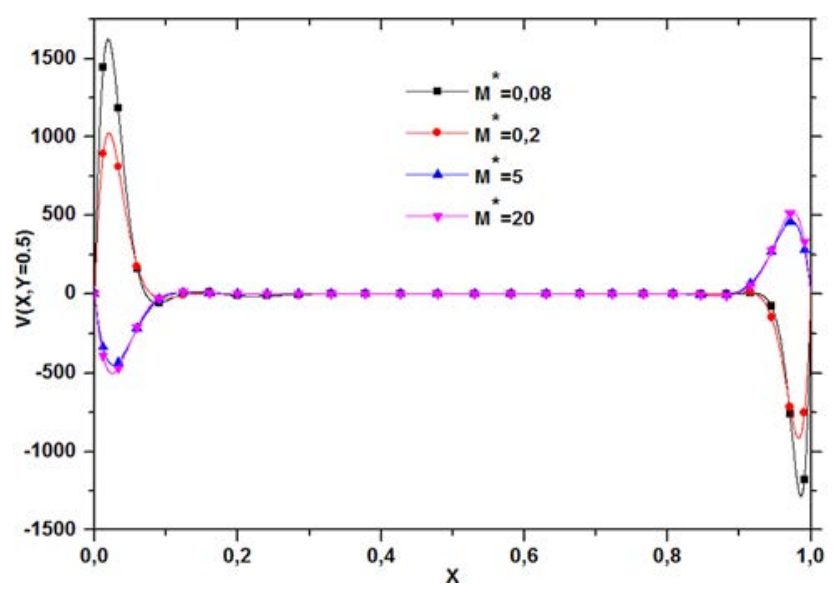

FIG. 14. Vertical velocity at the horizontal cross section at $Y=0.5$ for $R a=10^{6}$ and $L e=1$.

and horizontal velocity at the vertical cross section, respectively. It can be seen that the temperature (Fig. 13) and vertical velocity (Fig. 14) profiles are centro-symmetric with zero values at the cavity center. This symmetry is also found on the horizontal velocity (Fig. 15).

At this limit of $M^{*}, M^{*} \geq 0.105$, the variation in density and mass diffusivity [Figs. 9(a) and 9(b)] with respect to their reference values is no longer considerable. The maximum and minimum values of the horizontal and vertical velocities [Figs. 8(a) and 8(b)] approach increasingly indicating symmetry in their profiles.

For low $M^{*}$, higher temperature and concentration gradients are present in the region close to the lower part of the hot wall and top part of the cold wall as shown in Figs. 12(a) and 12(b) $\left(M^{*}=0.2\right.$ and 0.08$)$.

For a considerable $M^{*}$, higher temperature and concentration gradients are observed near the top part of the hot wall and near the lower part of the cold wall [Figs. 12(a) and 12(b) for $M^{*} \geq 4.33$ ], which indicates higher heat and mass transfer by convection and diffusion, the velocity gradients become steeper at both vertical sides of the

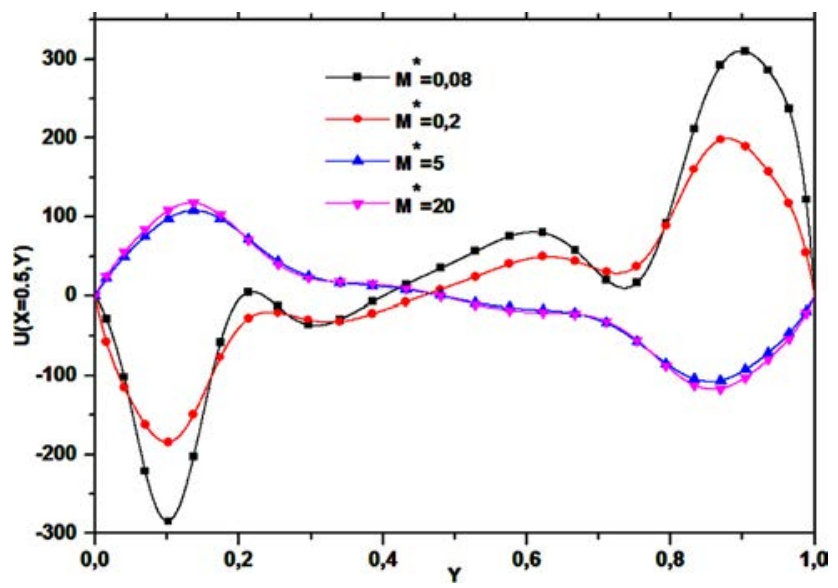

FIG. 15. Horizontal velocity at the vertical cross section at $X=0.5$ for $R a=10^{6}$ and $L e=1$. 
enclosure [Figs. 9(a) and 9(b) for $M^{*} \geq 4.33$ and $M^{*} \leq 0.2$ ]. These higher gradients in temperature and concentration cannot be observed if we use the Boussinesq model.

For very low values of $M^{*}, M^{*} \ll 0.105$, The symmetry seen in the previous case is broken due to the non-linear density and mass diffusivity variations whose role is gradually strengthened as the flow becomes more compressible.

On the other hand, for values of $M^{*} \leq 0.178(N \geq 18.95)$, the rate of change of mass diffusivity exceeds $10 \%$, [Fig. 6(b)], at which the thermodynamic pressure deviates by $8 \%$, (Fig. 7), compared to its value considered in the Boussinesq approximation $(\bar{P}=1)$, the symmetrical property of the solution is lost by $8 \%$.

\section{CONCLUSIONS}

Mathematical and numerical modeling of double-diffusive natural convection in an enclosure region is considered. The mathematical model has been formulated using the low Mach number formulation.

Numerical solution of the governing differential equations for the velocity, pressure, temperature, and concentration fields is obtained by using a finite volume technique. A power scheme was also used in approximating advection-diffusion terms. The SIMPLER algorithm with a staggered grid is employed to solve the coupling between pressure and velocity.

For large values of $M^{*}$, the low Mach number approximation approaches the Boussinesq Model for incompressible flows. Therefore, the solution schemes are usually based on numerical methods developed for incompressible flows.

Validation tests on the model have shown that the numerical simulation results in Boussniesq approximation are in good agreement compared with the numerical data available in literature.

Results show that the Boussinesq approximation is not sufficient to simulate double-diffusive natural convective flow when the buoyancy ratio exceeds the values between -6 and 27 and the flow becomes more compressible.

The changes in the thermal and dynamic fields introduced by the non-Boussinesq formulation are considerably larger than those for the aiding case.

If we take into account the variation of the mass diffusivity, it is preferable to use values of $N$, which must not exceed $N=19$.

Outside this range of $N$, all assumptions used to justify the Boussinesq approximation fail and a different modeling approach is required, one that accounts for realistic nonlinear fluid property variations.

It is interesting to note that there are a number of avenues which still remain to be explored or which have opened up during this work; we can mention the extension to the $3 \mathrm{D}$ modeling problem.

\section{ACKNOWLEDGMENTS}

The authors gratefully acknowledge the support of the Directorate General for Scientific Research and Technological Development (DGRSDT-Algeria).

\section{DATA AVAILABILITY}

The data that support the findings of this study are available within the article.

\section{REFERENCES}

'J. S. Turner, “Double diffusive phenomena," Annu. Rev. Fluid Mech. 6, 37-56 (1974).

${ }^{2}$ G. B. McFadden, S. R. Coriell, and R. F. Boisvert, "Double-diffusive convection with sidewalls," Phys. Fluids 28, 2716 (1985)

${ }^{3} \mathrm{~K}$. Ghorayeb and A. Mojtabi, "Double diffusive convection in a vertical rectangular cavity,” Phys. Fluids 9, 2339 (1997).

${ }^{4}$ R. Viskanta, T. L. Bergman, and F. P. Incropera, "Double diffusive natural convection," in Natural Convection: Fundamentals and Applications, edited by S. Kakac, W. Aung, and R. Viskanta (Hemisphere, Washington, DC, 1985), pp. 1075-1099.

${ }^{5} \mathrm{O}$. V. Trevisan and A. Bejan, "Combined heat and mass transfer by natural convection in a vertical enclosure," J. Heat Transfer 109, 104-112 (1987).

${ }^{6} \mathrm{M}$. Bouabid, N. Ghoudi, R. Bouabda, and M. Magherbi, "Irreversibility analysis for double diffusive convection flow of a gas mixture in a chamfered corners square enclosure filled with a porous medium," Arabian J. Sci. Eng. 45, 7499-7510 (2020).

${ }^{7}$ G. T. Kim Moo and H. Kwon, "Double-diffusive convection affected by conductive and insulating side walls during physical vapor transport of $\mathrm{Hg}_{2} \mathrm{Br}_{2}$," J. Korean Cryst. Growth Cryst. Technol. 30(3), 117-122 (2020).

${ }^{8}$ J. Serrano-Arellano, J. M. Belman-Flores, J. Xamán, K. M. Aguilar-Castro, and E. V. Macías-Melo, "Numerical study of the double diffusion natural convection inside a closed cavity with heat and pollutant sources placed near the bottom wall," Energies 13, 3085 (2020).

${ }^{9}$ Z.-C. Hu, S. H. Davis, and X.-R. Zhang, "Onset of double-diffusive convection in near-critical gas mixtures," Phys. Rev. E 99, 033112 (2019).

${ }^{10}$ I. C. Walton, "Double-diffusive convection with large variable gradients," J. Fluid Mech. 125, 123-135 (1982).

${ }^{11}$ C. Beghein, F. Haghighat, and F. Allard, "Numerical study of double diffusive natural convection in a square cavity," Int J. Heat Mass Transfer 35,833 (1992).

${ }^{12} \mathrm{~J}$. A. Weaver and R. Viskanta, "Natural convection in binary gases due to horizontal thermal and solutal gradients," J. Heat Mass Transfer 113, 14 (1991).

${ }^{13} \mathrm{~K}$. Chandra, "Linear instability in two-layer channel flow due to doublediffusive phenomenon," Phys. Fluids 32, 024102 (2020).

${ }^{14} \mathrm{H}$. Sun and G. Lauriat, "On the heat and mass transfer analogy for natural convection of non-dilute binary mixtures of ideal gases in cavities," C. R. Mec. 337, 141-149 (2009).

${ }^{15}$ H. Sun a, G. Lauriat a, D. L. Sun b, and W. Q. Tao, “Transient double-diffusive convection in an enclosure with large density variations," Int. J. Heat Mass Transfer 53, 615-625 (2010).

${ }^{16}$ J. A. Weaver and R. Viskanta, "Natural convection due to horizontal temperature and concentration gradients: 1. Variable thermophysical properties effects,” Int. J. Heat Mass Transfer 34(12), 3107-3120 (1991).

${ }^{17} \mathrm{~N}$. Laaroussi and G. Lauriat, "Conjugate thermosolutal convection and condensation of humid air in cavities," Int. J. Therm. Sci. 47, 1571-1586 (2008).

${ }^{18}$ J. H. Ferziger and M. Peric, Computational Methods for Fluid Dynamics (Springer, 2002).

${ }^{19}$ B. Ghernaout, S. Bouabdallah, A. Benchatti, and R. Bessaih, "Effect of the buoyancy ratio on oscillatory double-diffusive convection in binary mixture," Numer. Heat Transfer, Part A 66(8), 928-946 (2014).

${ }^{20}$ I. Sezai and A. A. Mohamed, "Double diffusive convection in a cubic enclosure with opposing temperature and concentration gradients," Phys. Fluids 12(9), 2210-2223 (2000).

${ }^{21}$ T. Nishimura, M. Wakamatsu, and A. M. Morega, "Oscillatory doublediffusive convection in a rectangular enclosure with combined horizontal temperature and concentration gradients," Int. J. Heat Mass Transfer 41(11), 1601-1611 (1998).

${ }^{22}$ T. R. Mahapatra, D. Pal, and S. Mondal, "Effects of buoyancy ratio on doublediffusive natural convection in a lid-driven cavity," Int. J. Heat Mass Transfer 57, 771-785 (2013).

${ }^{23}$ A. Bahloul, L. Kalla, R. Bennacer, H. Beji, and P. Vasseur, "Natural convection in a vertical porous slot heated from below and with horizontal concentration gradients,” Int. J. Therm. Sci. 43(7), 653-663 (2004). 
${ }^{24}$ Paolucci, S., "On the filtering of sound from the Navier-Stokes equations," Report No. SAND82-8257 (Sandia National Laboratories, Livermore, CA, 1982).

${ }^{25}$ S. Hamimid, M. Guellal, and M. Bouafia, "Numerical simulation of combined natural convection surface radiation for large temperature gradients," J. Thermophys. Heat Transfer 29(3), 636-645 (2015).

${ }^{26} \mathrm{M}$. Bouafia, S. Hamimid, and M. Guellal, "Non-Boussinesq convection in a square cavity with surface thermal radiation," Int. J. Therm. Sci. 96, 236-247 (2015).

${ }^{27}$ S. Hamimid, M. Guellal, and M. Bouafia, "Numerical study of natural convection in a square cavity under non-Boussinesq conditions," Therm. Sci. 20(5), 1509-1517 (2016).

${ }^{28}$ S. Hamimid, N. Rachedi, M. Guellal, and M. Bouafia, "Compressible coupled natural convection and volumetric radiation in a square cavity," J. Thermophys. Heat Transfer 32(3), 580-589 (2018).

${ }^{29} \mathrm{~W}$. Sutherland, "The viscosity of gases and molecular force," Philos. Mag. 36, 507-531 (1893).

${ }^{30}$ F. M. White, Viscous Fluid Flow (McGraw-Hill, 1974).
${ }^{31}$ T. R. Marrero and E. A. Mason, “Gaseous diffusion coefficients,” J. Phys. Chem. Ref. Data 1(1), 3-118 (1972).

${ }^{32}$ S. V. Patankar, Numerical Heat Transfer and Fluid Flow (McGraw-Hill, New York, 1980).

${ }^{33}$ J. Vierendeels, K. Riemslagh, and E. Dick, "A multigrid semi-implicit linemethod for viscous incompressible and low-Mach number flows on high aspect ratio grids," J. Comput. Phys. 154, 310-341 (1999).

${ }^{34} \mathrm{~V}$. Heuveline, "On higher-order mixed FEM for low Mach number flows: Application to a natural convection benchmark problem," Int. J. Numer. Methods Fluids 41, 1339-1356 (2003).

${ }^{35}$ C. Béghein, F. Haghighat, and F. Allard, "Numerical study of double-diffusive natural convection in a square cavity," Int. J. Heat Mass Transfer 35(4), 833-846 (1992).

${ }^{36}$ L. Koufi, Y. Cherif, Z. Younsi, and H. Naji, "Double-diffusive natural convection in a mixture-filled cavity with walls' opposite temperatures and concentrations," Heat Transfer Eng. 40, 1268-1285 (2019).

${ }^{37}$ D. D. Gray and A. Giorgini, "The validity of the Boussinesq approximation for liquids and gases," Int. J. Heat Mass Transfer 19, 545-551 (1976). 